COMECHINGONIA. Revista de Arqueología. Vol. 22, n² 2. Segundo semestre de 2018. ISSN: 0326-7911

\title{
LA ORIENTACIÓN DEL USHNU DE “EL SHINCAL DE QUIMIVIL” (CATAMARCA, ARGENTINA)
}

\section{THE ORIENTATION OF THE USHNU OF "EL SHINCAL DEL QUIMIVIL" (CATAMARCA, ARGENTINA)}

\author{
Gustavo Corrado ${ }^{1}$ y Sixto Giménez Benítez² \\ 1 Facultad de Ciencias Naturales y Museo, Universidad Nacional de La Plata. \\ Paseo del Bosque s/n, (1900) La Plata, Argentina, grrado@gmail.com; \\ 2 Facultad de Ciencias Astronómicas y Geofísicas, Universidad Nacional de La Plata. \\ Paseo del Bosque s/n, (1900) La Plata, Argentina, sixto@fcaglp.unlp.edu.ar
}

Presentado: 09/03/2018 - Aceptado: 10/12/2018

\section{Resumen}

Este trabajo expone una perspectiva arqueoastronómica del sitio inca "El Shincal de Quimivil" ubicado al sur del Tawantinsuyu (Catamarca, Argentina). Diversos autores han sostenido la existencia de orientas astronómicas relacionadas con este asentamiento. Este lugar se compone de numerosas estructuras edilicias como por ejemplo una plaza de $175 \mathrm{~m}$ de lado, un ushnu de $16 \mathrm{~m}$ de lado y un par de edificios kallanka dentro de la zona pública y varios otros vestigios de igual importancia. A su vez, se elevan sobre los laterales este y oeste de la plaza dos cerros aterrazados con imponentes escaleras. Sostenemos que es importante trabajar lugares alejados del Cuzco, de los cuales aún no se conocen detalles de interrelaciones ideológicas y sus correlatos materiales, que nos podrían dar pistas sobre los mecanismos, estrategias de apropiación y control Inca.

Palabras clave: inka - equinoccio medio temporal - arqueoastronomía - waka

\begin{abstract}
This work exposes an archaeoastronomic perspective of the Inca site "El Shincal de Quimivil" located south of the Tawantinsuyu (Catamarca, Argentina). Various authors have argued the existence of astronomical orientations related to this site. The site consists of many building structures such as a square $175 \mathrm{~m}$ on a side, one ushnu $16 \mathrm{~m}$ side and a pair of kallanka buildings in the public area and several other remains equally important. In turn, two terraced hills with imposing staircases rise on the east and west sides of the square. We believe it is important to work sites away from Cuzco, of which no details yet of ideological interrelationships and their material correlates, which could give us clues about the mechanisms, strategies of appropriation and control Inca known.
\end{abstract}

Key words: inka - temporary medium equinox - archaeoastronomy - waka 


\section{Introducción}

El ushnu es un concepto complejo, ya tratado en el mundo andino por varios autores (Hyslop 1990; Matos 1986; Meddens 1997; Zuidema 1980, entre otros), definido a partir de la existencia de plataformas simples o de más de un nivel ubicados en plazas o espacios públicos en la ciudad del Cuzco y en algunos de los principales centros administrativos y capitales provinciales del Tawantinsuyu, además de estar asociados a red vial incaica (Qhapaq Nan) (Guaman Poma 1980[1615]: 262). En general tienen formas rectangulares, de altura y orientación variable, en su mayoría presentan escaleras, terrazas, hornacinas y sistemas de drenaje subterráneo. El ushnu cumplía funciones de índole políticas, administrativas, religiosas y astronómicas (Gasparini y Margolies 1977; Hyslop 1990; Pino Matos 2004; Raffino et al. 1997; Zuidema 2011; Farrington 2013).

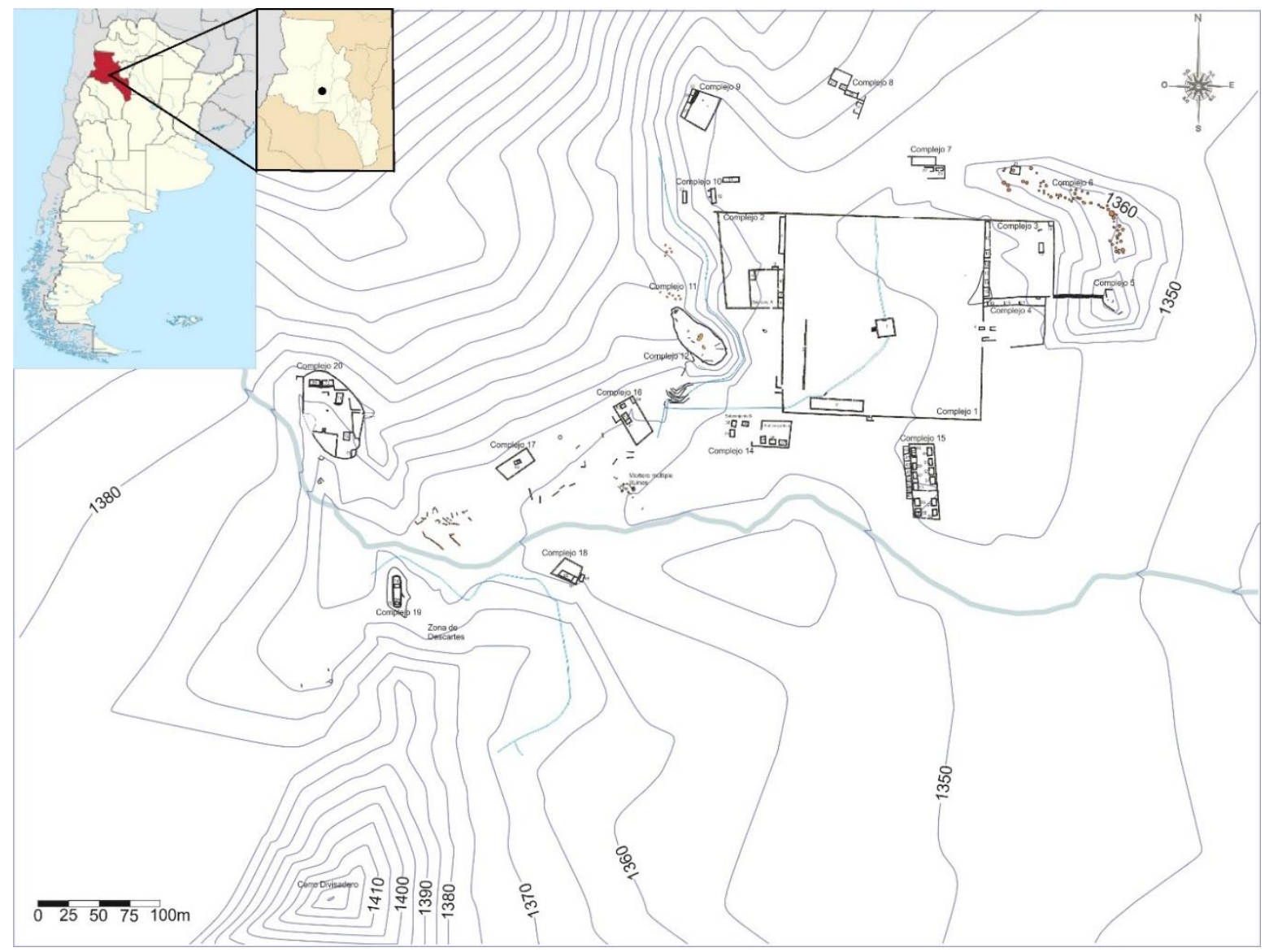

Figura 1. Plano completo de El Shincal de Quimivil (Giovannetti 2016). 
Para entender cómo el paisaje era escenario de interrelaciones sociales, es necesario identificar los aspectos geográficos sacralizados y sus vínculos espaciales. En este contexto, la arquitectura es la evidencia material donde se materializó parte de la ritualidad andina (Hastorf 2007: 97; Pino Matos y Moreano 2014).

En muchos pueblos andinos las montañas estaban vinculadas al culto de los antepasados (Leoni 2005; Vitry 2008). Es por esto, que algunas comunidades se creían descendientes de las deidades de las montañas, quienes representaban a los espíritus o bien eran sus moradas. En consecuencia, las montañas más elevadas y volcanes ocupaban un lugar primordial en sus creencias y rituales. Por ello existían toda una serie de prácticas de peregrinación ritual (Pino Matos y Moreano 2014).

Como observamos en el Cuzco, las relaciones sociales y sus divinidades se expresaron materialmente a través de la organización espacial y las construcciones monumentales (Julien 1999: 491), Lo que podría ser tomado como un modelo conceptual a replicar fuera de la capital cuzqueña en los espacios conquistados (Pino Matos y Moreano 2014).

En acuerdo con lo planteado por Pino Matos (Pino Matos, 2004), la organización del espacio manejada por los incas consideró como punto base, ejes o axis, asociados generalmente a centros simbólicos importantes, tales como el Qorikancha en el Cuzco y en las provincias probablemente este rol fue desempeñado por los ushnus (Pino Matos 2004: 306).

De este modo, en algunas capitales provinciales "El ushnu fue el primer punto a ubicar en el espacio, y basándose en él diseñaron el trazado de los asentamientos" (Pino Matos 2004: 306), como fue propuesto por Matos (Matos 1986) para el caso de Pumpu, en el altiplano de Chinchaicocha, Perú. Esto se relaciona con lo propuesto por Hyslop en cuanto a considerar al ushnu como centro de los asentamientos provinciales (Hyslop 1990), desempeñándose al mismo tiempo como un centro ceremonial y también como un centro físico, desde donde partían los alineamientos astronómicos que sirvieron para la organización espacial (Pino Matos 2004).

\section{El Shincal de Quimivil en la estructura del Tawantinsuyu}

El asentamiento que nos ocupa se ubica en la provincia de Catamarca, Argentina a unos $4 \mathrm{~km}$ del pueblo de Londres, entre los ríos Quimivil y Hondo, esta es una de las características que asemejaban a los "Nuevos Cusco" con el Cusco original. (Hylop 1990, Farrington 1999). 
Las primeras publicaciones que se refieren a trabajos de excavación arqueológica fueron realizadas por A. R. González (1966) quién estudió particularmente el sector de elite conocido como "Casa del Curaca" (complejo 20, Figura 1).

En la actualidad está aceptada la importancia regional de El Shincal en el mundo incaico del sur. Las características que definen a El Shincal como capital regional políticoadministrativa-ceremonial son, la gran plaza, kallankas sobre sus flancos, un ushnu central y elementos del paisaje que habrían funcionado como ejes directrices y representaciones similares a los identificados en el Cusco (Farrington 1999).

\section{El ushnu de El Shincal}

Este ushnu es la estructura de este tipo más grande al sur de Cochabamba (Raffino et al. 1997), en la cara oeste posee una escalera de acceso. La misma se compone de nueve peldaños que se elevan en total $1 \mathrm{~m}$, para finalizar en un vano simple de morfología trapezoidal que correspondería a la puerta de acceso hacia el interior de la plataforma (Giovannetti 2015). Los muros, son de tipo doble (entre 0.80 y $1 \mathrm{~m}$ de espesor) rellenos con barro para asentar. Todas las rocas con las cuales se construyó el ushnu, fueron canteadas para lograr caras planas y rectas. Al igual que el ushnu de Huánuco Pampa, presenta una tiana o banqueta interior (Raffino 2004). Se trata de una estructura alargada de $3 \mathrm{~m}$ de extensión por $0.80 \mathrm{~m}$ de espesor y $0.80 \mathrm{~m}$ de alto. Está colocada muy cerca de la pared norte de la estructura y se dispone de forma tal que su eje principal está en la dirección este-oeste. La posición del ushnu es casi central en relación a la plaza principal. Debemos mencionar que, si bien el sitio sufrió una reconstrucción de algunas de sus estructuras en los años recientes, el ushnu sólo fue intervenido levemente.

\section{El Shincal de Quimivil}

Dentro de la gran plaza, el ushnu no solamente se muestra como la representación de un escenario de ritos y ceremonias durante los eventos importantes, sino también, el eje espacial que marca alineamientos hacia puntos que seguramente jugaron un rol fundamental en el paisaje sagrado (Farrington et al. 2015: 58; Moyano et al. 2015: 258).

El ushnu y la plaza se encuentran enmarcados entre cuatro cerros que poseen evidencia antrópica. Los cerros este y oeste fueron aplanados artificialmente. En el cerro sur (Cerro La Loma Larga) se ha encontrado restos materiales anteriores a los incas (asociados a la Cultura Aguada) (González 1998). Al norte, otro cerro sugiere una importancia similar a los cerros este y oeste por su cantidad de vestigios en su superficie y alrededores (i.e. escalinata de acceso, construcciones circulares y rocas talladas). 


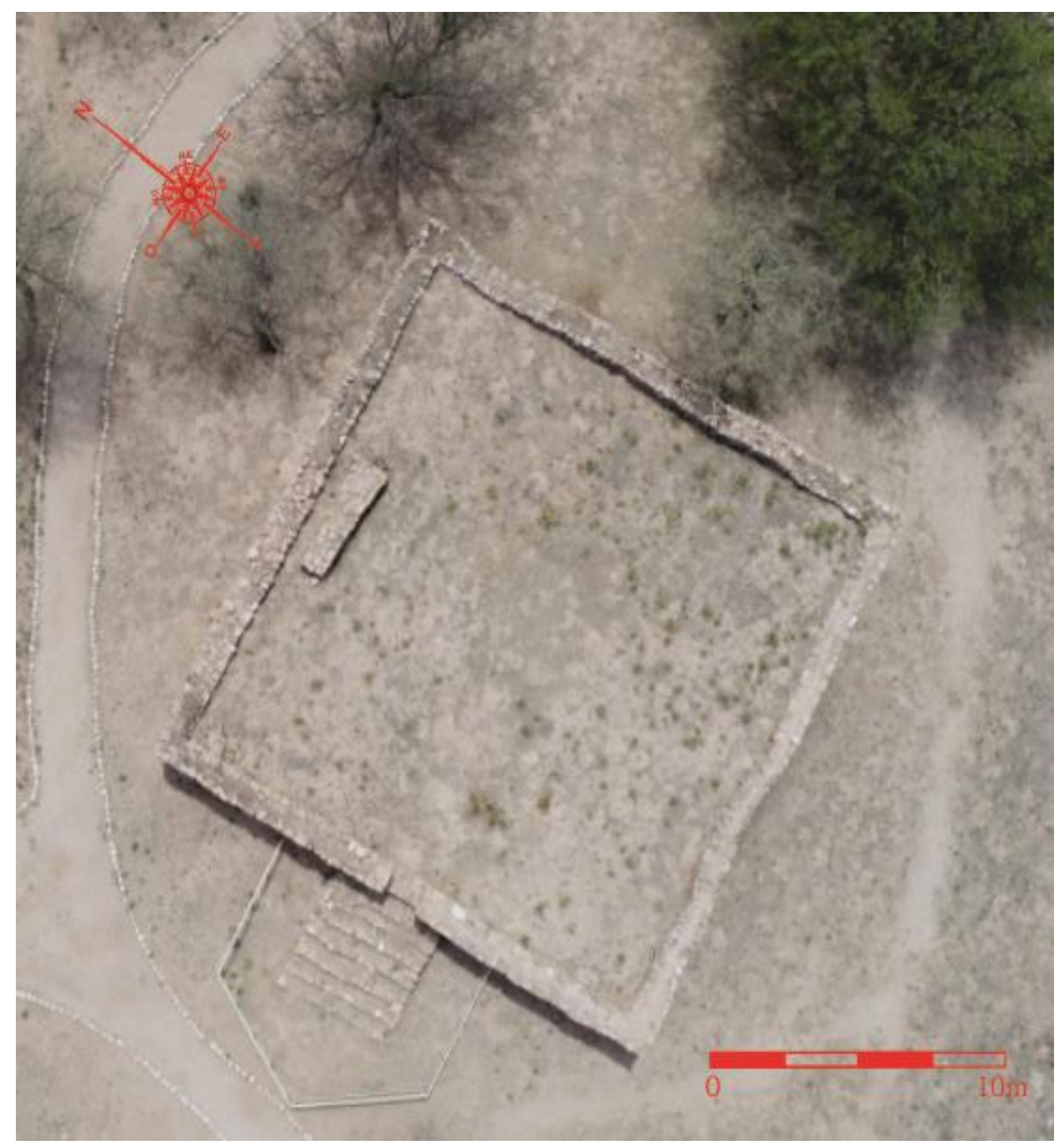

Figura 2. Vista en planta del Ushnu de El Shincal de Quimivil.

Farrington, en su trabajo sobre El Shincal en la década de 1990 (Farrington 1990) ubicó varios de los espacios sagrados en la geografía circundante alertando por primera vez sobre la importancia cultural de los cerros cercanos.

El Cerro Aterrazado este se dispone por fuera de la pared este de la gran plaza. Con una altura aproximada de $25 \mathrm{~m}$ con una escalinata de 103 peldaños que mira hacia la plaza.

En la cima de este cerro encontramos un semicírculo de piedra que podría identificarse como waka (Figura 3). 


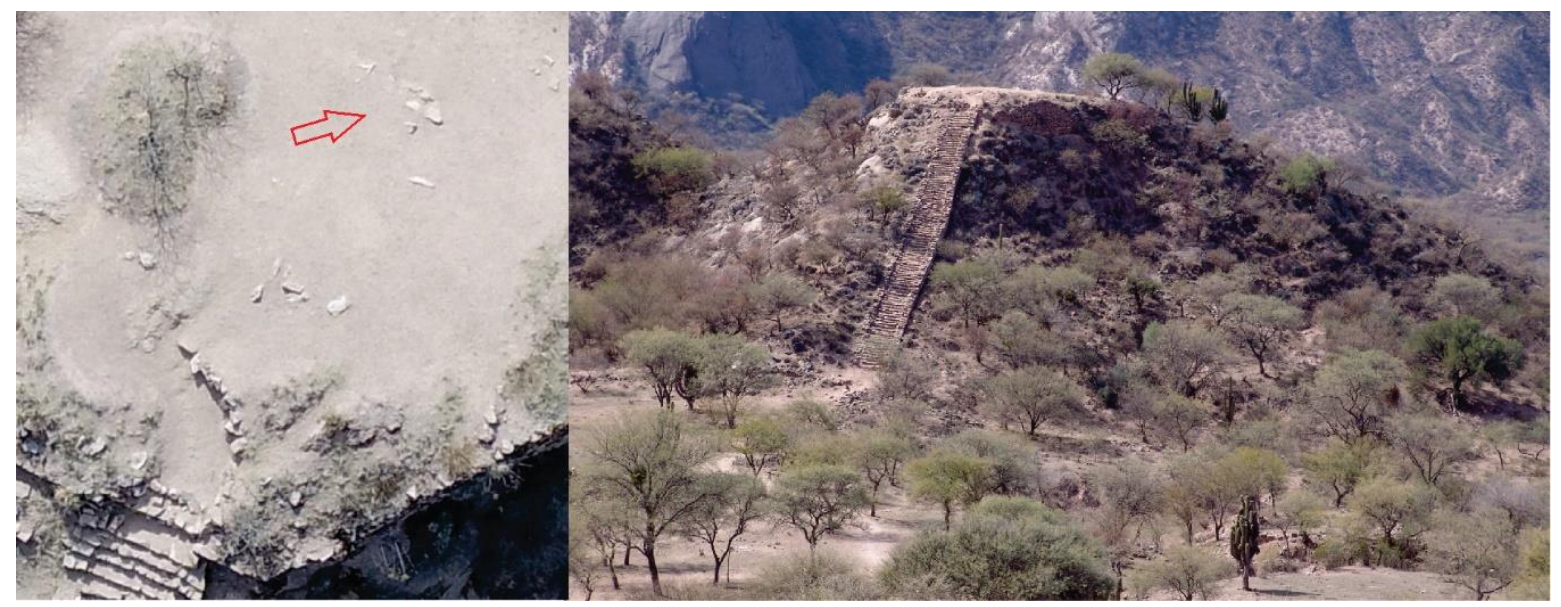

Figura 3. Izquierda: Vista en planta donde se observa el semicírculo de piedras. Derecha: Vista del Cerro donde se puede observar la escalera.

El cerro oeste posee una angosta escalera zigzagueante de más de 60 peldaños. Una vez en la cima del cerro encontramos cinco rocas, afloramientos graníticos propios del cerro, donde se aprecia su forma irregular, conservando su forma natural.

En este cerro encontramos manifestaciones antrópicas semejantes al cerro oriental, como dos rocas trabajadas y morteros. El cerro posee un muro muy bien construido que recubre toda la ladera del mismo realizando cambios de dirección para marcar una morfología recortada también como zig-zag. Este muro nos muestra una forma de apropiación de estilo cusqueño en su diseño arquitectónico, similar a lo observado en Saqsaywaman y Wata en Cusco, Incallajta en Bolivia (Hyslop 1990) y sitio incaico de Turi en Chile (Berenguer y Salazar 2017).

En el cerro oeste, las rocas que se despliegan en su cima, posiblemente conformaron una waka. No sabemos, si previo a la llegada de los incas se veneraba este cerro, aunque sí se sabe que fue tomado por estos conquistadores como un espacio especial (Giovannetti 2016: 85).

Ambos cerros fueron aplanados, sus laderas amuralladas y poseen escaleras construidas con cientos de rocas canteadas. Además de otorgarles un aspecto imponente, los coloca en una situación prácticamente única entre los sitios arqueológicos inca del noroeste argentino (Corrado y Giménez Benítez 2015; Corrado et al. 2014; Corrado et al. 2016). 


\section{Datos observacionales}

En este trabajo realizamos observación en el lugar, de los equinoccios de marzo de 2015 y septiembre 2016, desde el ushnu, con el fin de obtener alturas y azimuts de los puntos importantes de los cerros este y oeste y la relación del ushnu con el movimiento del sol. Para ello utilizamos un tándem Suunto que posee brújula (exactitud $1 / 3^{\circ}$ ) y clinómetro (exactitud $\left(1 / 4^{\circ}\right)$. Las correcciones magnéticas para las fechas de observación fueron obtenidas del National Geophysical Data Center [NGDC]. Además, los planos del sitio fueron realizados con una Estación Total Pentax R-315NX (precisión angular + / - 5*). La georreferenciación se realizó utilizando un GPS Diferencial Thales Mobile Mapper (Giovannetti et al. 2013).

La declinación magnética en El Shincal para el 21 de marzo de 2015 era de $4^{\circ} 09^{\prime}$ W variando $0^{\circ} 18^{\prime} \mathrm{W}$ por año (con un error -uncertainty- de $0,36^{\circ}$ ) y para el 20 de septiembre de 2016 era de $4^{\circ} 22^{\prime} \mathrm{W}$ variando $0^{\circ} 11^{\prime} \mathrm{W}$ por año (con un error de $0,22^{\circ}$ ).

Una vez obtenidos los datos hemos realizado el cálculo de las declinaciones correspondientes utilizando las ecuaciones de cambio de coordenadas del sistema horizontal al ecuatorial celeste (Baume 2014) y utilizando el modelo de Bennett, para la corrección por refracción (Bennett 1982).

Como ya hemos mencionado el ushnu se encuentra dentro de una plaza rectangular, pero no comparte la misma orientación, el ushnu está rotado con respecto a la plaza unos $4^{\circ}$. Y la plaza está orientada cardinalmente (con un error de $1^{\circ}$ en promedio).

Hemos reconocido que la orientación del ushnu responde a dos causas:

a) La línea que pasa por el círculo de piedras sobre el Cerro Este $\left(\mathrm{A}=84^{\circ} 50^{\prime}\right)$ y la roca waka del Cerro Oeste $\left(A=265^{\circ} 10^{\prime}\right)$, ambos medidos desde el ushnu (Figura 5). Ambos datos corregidos por desviación magnética.

b) La salida del sol en los equinoccios de marzo y septiembre, pero la orientación equinoccial no correspondería al equinoccio astronómico $\left(\delta\right.$ del Sol $\left.=0^{\circ}\right)$ como afirman algunos autores (Moyano 2013, 2016; Moyano et al. 2016; Farrington 2015), sino al equinoccio medio temporal (Ruggles 1997; González García y Belmonte 2006) (Figura 4). 


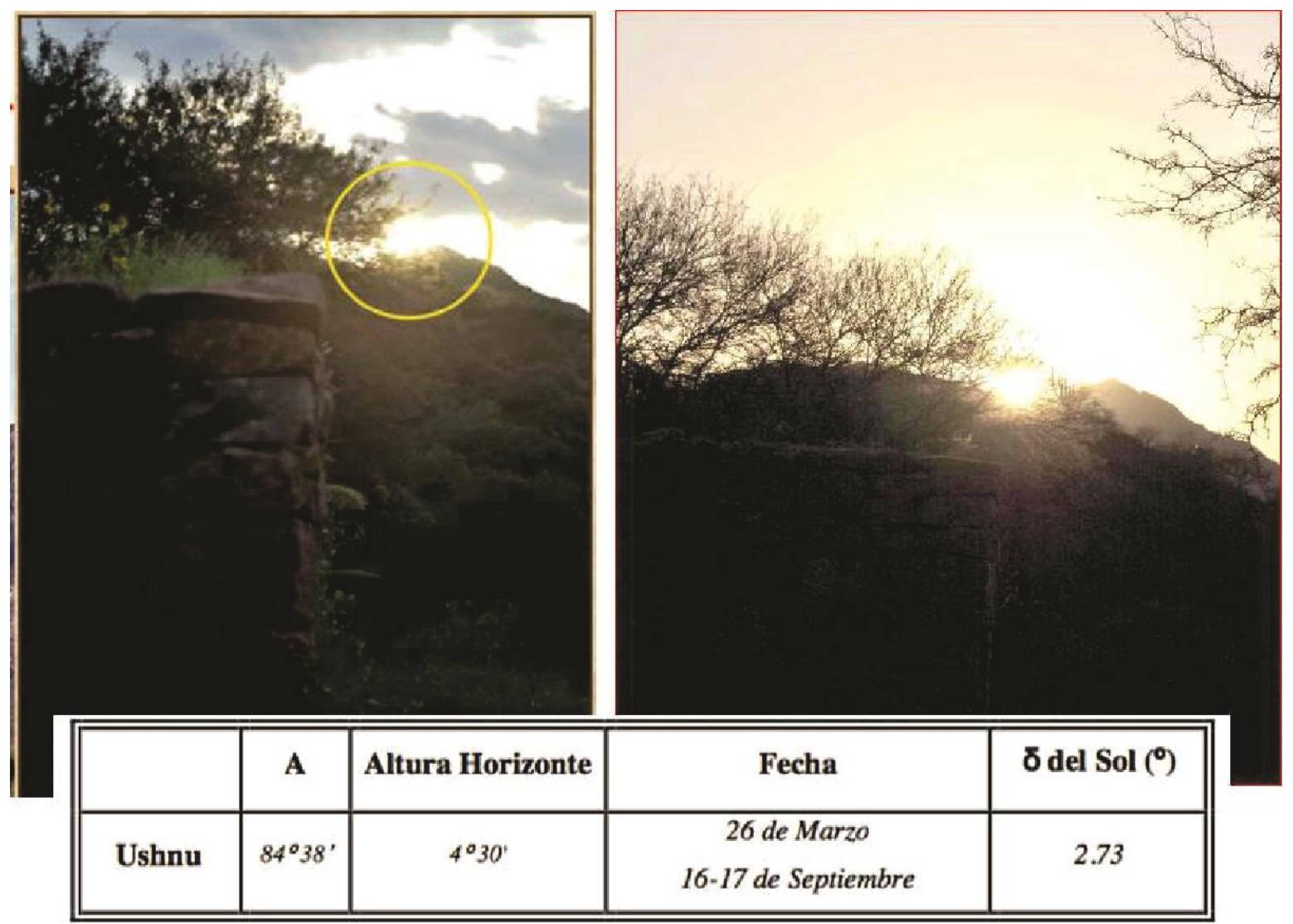

Figura 4. Izquierda: Salida del Sol en el equinoccio astronómico de marzo de 2015. Derecha: Salida del Sol el 18 de septiembre de 2016.

Tabla: datos de la orientación del ushnu, A, corregido por desviación magnética.

La construcción y ubicación del ushnu podría responder a la idea de los incas de resignificar los elementos importantes del paisaje preexistente de los territorios conquistados, reutilizando los elementos potencialmente sagrados de la topografía local (Giovannetti 2015; Moyano 2010; Corrado y Giménez Benítez 2015; Corrado et al. 2014; Corrado et al. 2016). En el caso de El Shincal la alineación del ushnu con los cerros este y oeste responderían a esta idea en la cual detectamos que a la orientación hacia los cerros (que encontramos en otros sitios inca) se le superpone la orientación a la salida del sol en una fecha solar importante. Este hecho sería similar a lo que ocurre en Pumpu, donde el eje del ushnu, además de corresponder a la orientación hacia cerros importantes del paisaje local (que son wakas), se superpone con la salida del sol el día del paso cenital en el lugar (Pino Matos y Moreano 2014). 


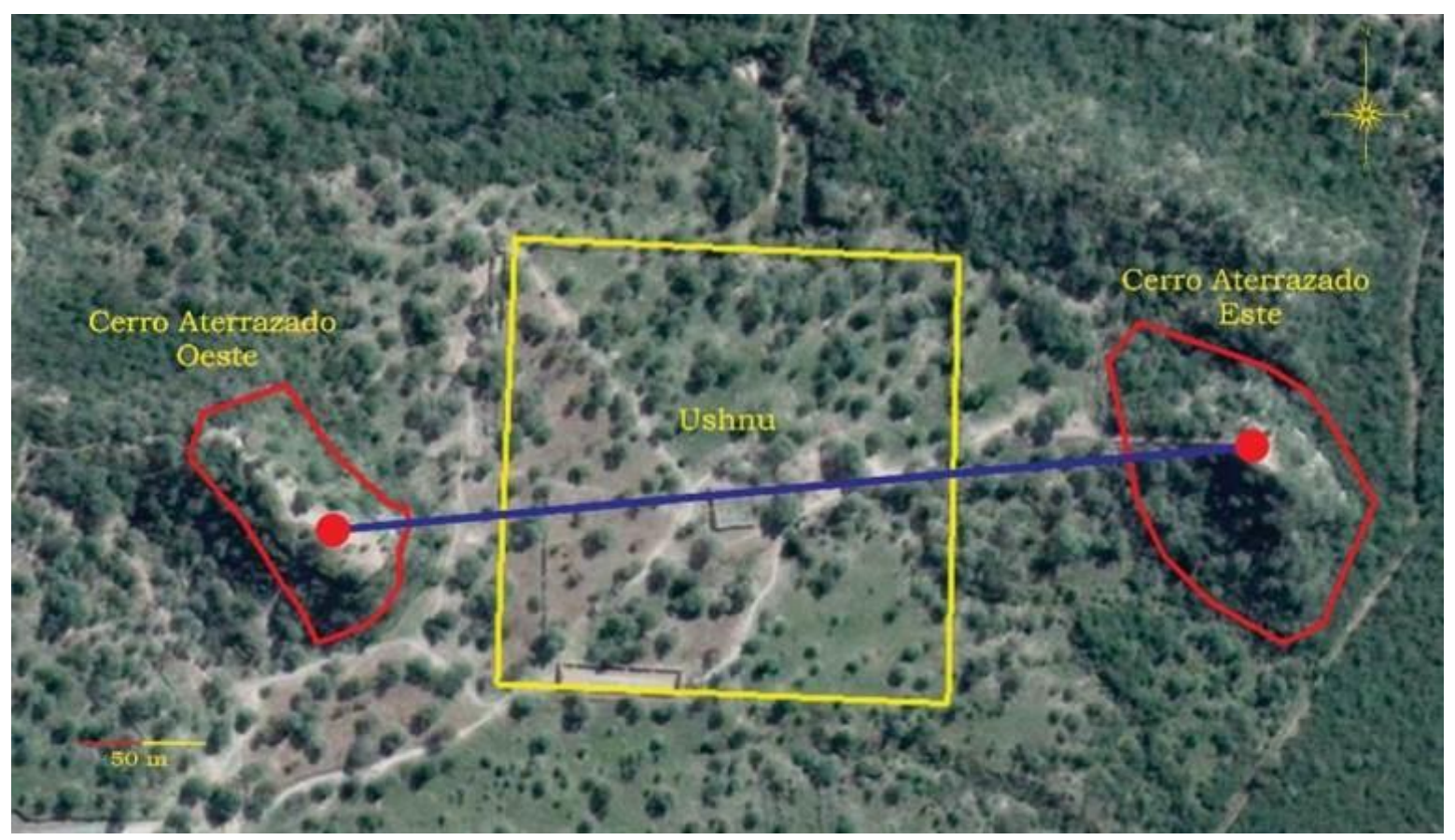

Figura 5. Orientación del ushnu respecto a los cerros este y oeste.

\section{Conclusión}

Es de suma importancia evaluar el grado de integración de los paisajes locales dentro de la cosmovisión inca en un sitio que se encuentra casi al extremo sur del Tawantinsuyu. Parte del control territorial consistía en dominar las fuerzas que emanaban de cada geografía particular a partir, también, del control de sus correspondientes wakas, que podían materializarse como rocas, montañas o ríos.

Después de analizar la información obtenida en el ushnu de El Shincal de Quimivil, vemos que su orientación no sería al equinoccio astronómico, sino que hay un compromiso entre la orientación al equinoccio medio temporal, y dos wakas en los cerros este y oeste. Cabe señalar que estas no son las únicas wakas del sitio, pero son las que se relacionan con la orientación del ushnu.

Encontramos en ushnu de El Shincal, las mismas ideas que vemos en sitios como Pumpu, en altiplano de Chichaicocha en Perú (Pino Matos y Moreano 2014) en la cual se relaciona el paisaje local con fenómenos solares importantes. 


\section{Bibliografía citada}

Baume, G.

2014 La Esfera Celeste. Universidad Nacional de La Plata, La Plata.

Bennet, G.

1982 The calculation of astronomical refraction in marine navigation. Journal of Navigation 35: 255-259.

Berenguer, J. y D. Salazar

2017 Territorialización del modelo minero inkaico en el Río Salado: una aglomeración productiva entre Lípez y San Pedro de Atacama. Boletín del Museo Chileno de Arte Precolombino 22(1): 51-69.

Corrado, G. y S. Giménez Benítez

2015 El Ushnu, como organizador del espacio sagrado en El Shincal (Catamarca, Argentina). Trabajo presentado en la Tercera Jornada de Astronomía Cultural y La Cuarta Escuela Interamericana de Astronomía Cultural, Brasil.

Corrado, G.; Giovannetti, M.; Gimenez, S.; Pino Matos, J. y W. Moreano Montalván 2014 El Shincal: paisaje ritual y astronomal. Trabajo presentado en las II Jornadas Interamericanas de Astronomía Cultural. INAH, ENAH, México.

Corrado, G.; Giovannetti, M.; Moreano Montalván, W. y S. Giménez

2016 Las plazas Inka como escenarios de instituciones festivas calendarizadas: una aproximación comparativa de los espacios públicos ortogonales del Shincal de Quimivil (Collasuyu) y Huánuco Pampa (Chinchaysuyu)". Trabajo presentado en el III Congreso Nacional de Arqueología. Lima.

Farrington, I.

1999 El Shincal: un Cusco del Kollasuyu. Actas del XII Congreso Nacional de Arqueología Argentina, tomo I, pp. 53-62. La Plata.

2013 Cusco, Urbanism and Archaeology in the Inka World. University Press of Florida. Gainesville.

Farrington, I.; Moyano, R. y. G. Díaz

2015 El Shincal de Quimivil. La importancia de los estudios arqueoastronómicos. En Una Capital Inka al Sur del Kollasuyu: El Shincal de Quimivil, R. Raffino, A. Iácona, R. Moralejo, D. Gobbo y M. Couso (eds.), pp. 41-61. Fundación de Historia Natural Félix de Azara, Buenos Aires. 
Gasparini, G. y L. Margolies

1977 Arquitectura Inca. Universidad Central de Venezuela, Caracas.

Giovannetti, M.

2015 Agricultura, Regadí y Molienda en Una Capital Inkaica: Los Sitos El Shincal y Los Colorados, Noroeste Argentino. BAR S2702, Oxford.

2016 Fiestas y Ritos Inka en El Shincal de Quimivil. Punto de Encuentro, Buenos Aires.

Giovannetti, M.; Cochero, G.; Spina, J.; Corrado, G.; Valderrama, M.; Aljanati, L. y E. Ferraris 2013 El Shincal de Quimivil, La Capital Ceremonial Inka del Noroeste Argentino. Editorial QuireQuire, La Plata.

González, A.

1966 Las Ruinas del Shincal. Actas del Primer Congreso de Historia de Catamarca, tomo II, pp. 15-28. Junta de Estudios Históricos de Catamarca, San Fernando del Valle de Catamarca. 1998 Cultura La Aguada. Arqueología y Diseños. Filmediciones Valero, Buenos Aires.

González García, A. y J. Belmonte

2006 Which equinox? Archaeoastronomy. The Journal for Astronomy in Culture 20: 95-105.

Guaman Poma de Ayala, F.

1980 [1615] El Primer Nueva Crónica y Buen Gobierno. Editorial Siglo Veintiuno, México.

Hastorf, C.

2007 Archaeological andean rituals: performance, liturgy, and meaning. En The Archaeology of Ritual, E. Kyriakidis (ed.), pp. 77-107. Cotsen Institute of Archaeology, University of California, Los Angeles.

Hyslop, J.

1990 Inca Settlement Planning. University of Texas Press, Austin.

Julien, C.

1999 El Tawantinsuyu. En Historia de América Andina. Las Sociedades Aborígenes, L. Lumbreras (ed.), vol. 1, pp. 435-497. Universidad Andina Simón Bolívar, Quito

Leoni, J.

2005 La veneración de montañas en los Andes preincaicos: el caso de Nawinpukyo (Ayacucho, Perú) en el Período Intermedio Temprano. Chungara 37(2): 151-164. 
Matos, R.

1986 El ushnu de Pumpu. Cuicuilco 18: 45-61.

Meddens, F.

1997 Function and meaning of the usnu in Late Horizon Peru. Tawantinsuyu 3: 5-14.

Moyano, R.

2010 El ushnu y la astronomía de horizonte en Viña del Cerro. Chungara 42(2): 419-432.

2013 La Luna como Objeto de Estudio Antropológico: El Ushnu y la Predicción de Eclipses en Contextos Incas del Collasuyu. Tesis de Doctorado. ENAH, México.

2016 La observación de la Luna en contextos incas del Collasuyu. En Perspectivas Etnográficas e Históricas sobre as Astronomías, P. Faulhaber y L. Borges (eds.), pp. 128-150. Museu de Astronomia e Ciências Afins - MAST, Rio de Janeiro.

Moyano, R.; Díaz, G.; Farrington, I.; Moralejo, R.; Cuoso, G. y R. Raffino

2015 Arqueoastronomía en El Shincal de Quimivil: análisis preliminar de un sitio inca en la franja del lunisticio mayor al sur. En Arqueología y Paleontología de la Provincia de Catamarca, V. Rodríguez y M. López (eds.), pp. 249-260. Fundación de Historia Natural Félix de Azara, Buenos Aires.

National Geophysical Data Center

2015 http:/ / www.ngdc.noaa.gov/geomag/

2016 http://www.ngdc.noaa.gov/geomag/

Pino Matos, J.

2004 El ushnu inka y la organización del espacio en los principales tampus de los wamani de la Sierra Central del Chinchaysuyu. Chungara 36(2): 306-311.

Pino Matos, J. y W. Moreano

2014 El ushnu, el qhapaq ñan y las wacas en el Altiplano del Chinchaycocha. Una aproximación a las estrategias de apropiación y control territorial Inca, desde la lectura de los paisajes rituales y la astronomía. Haucaypata 8: 60-90.

Raffino, R.

2004 El Shincal de Quimivil. En El Shincal de Quimivil, R. Raffino (ed.), pp. 22-43. Editorial Sarquís, San Fernando del Valle de Catamarca.

Raffino, R.; Gobbo, D.; Vázquez, R.; Capparelli, A.; Montes V.; Iturriza, D.; Deschamps, C. y M. Mannasero

1997 El ushnu de El Shincal de Quimivil. Tawantinsuyu 3: 22-39. 
Ruggles, C.

1997 Whose equinox? Archaeoastronomy 22: S45-50.

Vitry, C.

2008 Los espacios rituales en las montañas donde los inkas practicaron sacrificios humanos. En Paisagens Culturais. Contrastes Sul-americanos. C. Terra y R. Andrade (eds.), pp. 47-65. Universidade Federal do Rio de Janeiro, Escola de Belas Artes, Rio de Janeiro.

Zuidema, T.

1980 El ushnu. Revista de la Universidad Complutense 28: 17-362.

2011 El Calendario Inca. Tiempo y Espacio en la Organización Ritual del Cusco, la Idea del Pasado. Fondo editorial del Congreso del Perú, Fondo editorial de la Pontificia Universidad Católica del Perú, Lima. 
